\title{
Electrocaloric Effect in Sodium Bismuth Titanate Based Ferroelectric Composites
}

\author{
A. Rajani Malathi ${ }^{\star}$ G. S. Kumar, G. Prasad
}

Mixed system of (1-x) $\mathrm{Na}_{0.5} \mathrm{Bi}_{0.5} \mathrm{TiO}_{3}+(\mathrm{x}) \mathrm{SrTiO}_{3}$ (NBT-ST) where $\mathrm{x}=0.075,0.125,0.150,0.200$ ferroelectric ceramic materials are synthesized using modified pechini method. These optimized compositions are used for study of polarization versus electric field (P-E) loops. The ferroelectric response of the samples is investigated as a function of temperature. Remnant polarization of exhibited loops observed to decrease with increasing temperature. PE data is used to estimate the electrocaloric properties. Maximum entropy change, full width at half maximum, relative cooling power is obtained from theoretical calculations.

\section{Introduction}

Perovskite ferroelectric materials exhibit electrocaloric effect (ECE) which is associated with entropy change in the material during application or removal of field. This electrocaloric effect is used for ferroelectric refrigeration. Commonly, the change of entropy between lowtemperature ferroelectric phase with ordered dipoles and high-temperature paraelectric phase without ordered dipoles is involved in ECE process and remarkable ECE always occurs near Curie temperature. The dipole-ordered state can be enhanced by decreasing temperature or enhancing applied electric field. Hence, the entropy drops and the ferroelectric material releases heat during application of field, while the entropy rises, and the material absorbs heat during withdrawal of field. Relaxor ferroelectrics could be attractive for ECE applications [1-4].

NBT is a promising candidate for ferroelectric and piezoelectric applications. It belongs to perovskite family. It undergoes various structural as well as phase transitions at different temperatures [5]. Compositional modifications in NBT results better properties. Addition of $\mathrm{SrTiO}_{3}$ to NBT shows better ferroelectric and piezoelectric properties [6]. Several authors have studied the electrocaloric effect on NBT based solid solutions. Yang Bai et. al., [7] reported abnormal electrocaloric effect of $\mathrm{Na}_{0.5} \mathrm{Bi}_{0.5} \mathrm{TiO}_{3}-\mathrm{BaTiO}_{3}$ lead-free ferroelectric ceramics above room temperature. Harberg et. al., [8] studied the electrocaloric effect in $\mathrm{Na}_{1 / 2} \mathrm{Bi}_{1 / 2} \mathrm{TiO}_{3}-\mathrm{SrTiO}_{3}-\mathrm{PbTiO}_{3}$ solid solutions. There are

Department of Physics, Osmania University, Hyderabad 500 007, India

${ }^{*}$ Corresponding author:

rajanimalathi27@gmail.com

DOI: 10.5185/amlett.2021.111676 several applications for this technology, but the most intuitive application would be to cool computer chips and devices by employing refrigeration cycle [9].

In view of this, an effort has been made to investigate the PE loops, electrocaloric behavior, entropy change and relative cooling power of $(1-\mathrm{x}) \mathrm{Na}_{0.5} \mathrm{Bi}_{0.5} \mathrm{TiO}_{3}+(\mathrm{x})$ $\mathrm{SrTiO}_{3}$ (NBT-ST) where $\mathrm{x}=0.075,0.125,0.150,0.200$ samples and results of such an investigation are presented in this paper.

\section{Experimental}

\section{Material synthesis}

NBT and ST samples are synthesized using modified pechini method. For the preparation of NBT, the stoichiometric proportion of $\mathrm{NaNO}_{3}$ (SD fine 99.5\%), Bi $\left(\mathrm{NO}_{3}\right)_{3}$ (SD fine 99.5\%), Ti (100 mesh, Aldrich 99.7\%), $\mathrm{H}_{2} \mathrm{O}_{2}$ (30\%, SD fine) and Ammonia solution (25\% AR grade, SD fine) are taken. Initially the required proportions of constituents are dissolved in water. The Ti metal powder is added to a solution containing $70 \mathrm{ml}$ of $\mathrm{H}_{2} \mathrm{O}_{2}$ and $30 \mathrm{ml}$ of Ammonia at $0^{\circ} \mathrm{C}$. Under constant stirring yellow gel is obtained after $12 \mathrm{~h}$. Then, this solution is added to the previously prepared aqueous solution of $\mathrm{Na}$ and $\mathrm{Bi}$ nitrates in appropriate quantities. Later the citric acid is added to this solution in the molar ratio of $2: 1$ so that citrate is formed. The $\mathrm{P}^{\mathrm{H}}$ of the resultant solution is adjusted between 6 to 7 by adding Ammonia and the solution is heated. After 48 hrs, thick viscous liquid is obtained. At this stage ethylene glycol is added in the molar ratio1:1.2 of the citric acid to the ethylene glycol. The mixture is heated at $180^{\circ} \mathrm{C}$ for 5-6 hrs. A black precursor solid is obtained. The precursor powder obtained is ground and calcined at various temperatures required for the phase formation. Similarly for the preparation of $\mathrm{SrTiO}_{3}$, the initial compounds are $\mathrm{Sr}\left(\mathrm{NO}_{3}\right)_{2}$ (SD fine 99.5\%), Ti (100 mesh 
Aldrich 99.7\%), $\mathrm{H}_{2} \mathrm{O}_{2}(30 \% \mathrm{SD}$ fine) and Ammonia solution (25\% AR grade SD fine) taken the remaining procedure for preparation is similar as NBT.

The calcined powders of component compounds of the composite are mixed in molar ratio as required for synthesizing composite materials. The powder is ground for 6 hours to obtain a homogeneous mixture. The mixture obtained is pressed into pellets of about $10 \mathrm{~mm}$ diameter and $2 \mathrm{~mm}$ thickness using a hydraulic press. About $2 \%$ of Poly vinyl alcohol (PVA) binder is added during the process of pelletization. The pellets are sintered in the temperature range of $1160-1190^{\circ} \mathrm{C}$ (depending on the composition) for $5 \mathrm{~h}$ for densification. The sintered samples are annealed at $800^{\circ} \mathrm{C}$ for $4 \mathrm{~h}$. These samples are electroded with silver paint on both sides for electrical contact. From here after the samples (1-x) $\mathrm{Na}_{0.5} \mathrm{Bi}_{0.5} \mathrm{TiO}_{3}+(\mathrm{x}) \quad \mathrm{SrTiO}_{3}$ (NBT-ST) where $\mathrm{x}=0.075,0.125,0.150,0.200$ are named as NBSTA, NBSTB, NBSTC and NBSTD.

\section{Characterization}

Polarization of the samples is observed by using custom built automatic P-E loop tracer of Marine India ltd. working at $50 \mathrm{~Hz}$ frequency. The measurement is carried out from room temperature to $150^{\circ} \mathrm{C}$ at an interval of $25^{\circ} \mathrm{C}$, with constant heating rate of $5^{\circ} \mathrm{C} / \mathrm{min}$.

\section{Results and discussion}

\section{P-E LOOPS}

The polarization vs. electric field hysteresis loops of NBSTA-NBSTD at room temperature are shown in Fig. 1. Generally, the PE loops were resulted from the electro-mechanical interaction between the polar and nonpolar regions, which coexisted in the BNT-based ceramics [10]. The values of remanent polarization (Pr) and coercive field (Ec) derived from the hysteresis loops. As the ST content increase in the samples, remnant polarization increases and coercive field decreases. The remanent polarization of the sample is observed to increased upto $100^{\circ} \mathrm{C}$ and further increase in temperature decrease in $\mathrm{Pr}$ is observed (not shown in figure). Between $100^{\circ} \mathrm{C}-180^{\circ} \mathrm{C}$, Ferroelectric to antiferroelectric transition observed in NBT sample [11]. This particular transition makes the sample for polarization decrease after $100^{\circ} \mathrm{C}$. Coercive field of the sample is decreased with increase in temperature. The shapes of loops become slim with temperature. Whereas in NBT based samples polarization of the samples is increased with temperature till it reaches the temperature $200^{\circ} \mathrm{C}$, these samples can exhibit ferroelectric behavior upto this temperature. Above this temperature they become antiferroelectric. While approaching higher temperatures ferroelectricity is decreased and antiferroelectricity is increased. Antiferroelectric materials also exhibit polarization with the application of external field. $P_{r}$ and $E_{c}$ values of NBSTA-NBSTD samples are listed in Table 1.
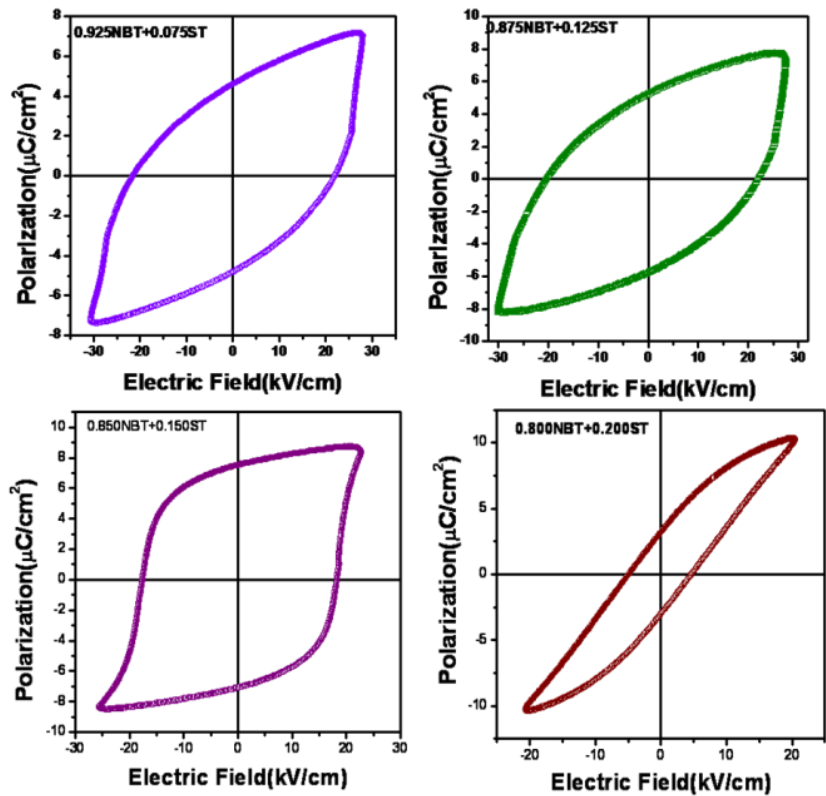

Fig. 1. Polarization vs Electricfield loops for NBSTA, NBSTB, NBSTC and NBSTD at room temperature.

Table 1. Remanence $\left(\mathrm{P}_{\mathrm{r}}\right)$, Coercivity $\left(\mathrm{E}_{\mathrm{c}}\right), \mathrm{P}_{\max }$, and Maximum Field $\left(\mathrm{E}_{\max }\right)$ values for NBST samples.

\begin{tabular}{lccccc}
\hline Sample & $\begin{array}{c}\mathbf{P r} \\
\left(\boldsymbol{\mu} \mathbf{C} / \mathbf{c m}^{2}\right)\end{array}$ & $\begin{array}{c}\mathbf{E}_{\mathbf{c}} \\
(\mathbf{k V / c m})\end{array}$ & $\mathbf{P}_{\max }$ & $\mathbf{P r}_{\mathbf{P}} \mathbf{P}_{\max }$ & $\begin{array}{c}\mathbf{E}_{\max } \\
(\mathbf{k V / c m})\end{array}$ \\
\hline NBSTA & 4.708 & 25.565 & 7.259 & 0.648 & 29.387 \\
NBSTB & 5.486 & 21.162 & 8.013 & 0.684 & 28.748 \\
NBSTC & 7.295 & 17.922 & 8.625 & 0.845 & 24.134 \\
NBSTD & 3.109 & 4.794 & 10.341 & 0.300 & 20.535 \\
\hline
\end{tabular}

\section{Electrocaloric effect}

In general, ECE is effectively observed in ferroelectric materials which are capable of generating large polarization change and show large entropy variation associated with the polarization change [12]. So, the present samples have been considered as appreciable candidates to observe electrocaloric effect. Many experimental methods are found in the literature to observe the electrocaloric effect in the NBT based samples. But theoretical approaches are getting their importance to evaluate this effect. In fact, Hamad used the similar theoretical equations to evaluate the electrocaloric as well as magnetocaloric effect in many samples [13].

According to this theoretical model, the variation of polarization with temperature which is shown in Fig. 2 is simulated by

$$
P=\left(\frac{P_{i}-P_{f}}{2}\right)\left[\tanh \left(A\left(T_{C}-T\right)\right)\right]+B T+C
$$

where $P_{i}$ is an initial value of polarization at ferroelectricparaelectric transition and $\mathrm{P}_{\mathrm{f}}$ is a final value of polarization at ferroelectric-paraelectric transition. 


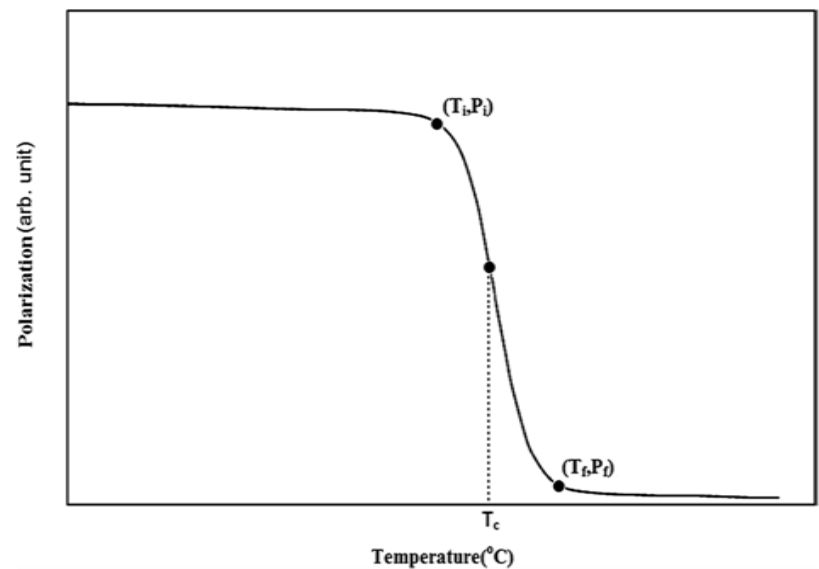

Fig. 2. Dependence of polarization as a function of temperature.

$$
A=\frac{2\left[B-\left.\frac{d P}{d T}\right|_{T=T_{C}}\right]}{P_{i}-P_{f}}
$$

B is polarization sensitivity $\frac{d P}{d T}$ at ferroelectric state before transition, $\left.\frac{d P}{d T}\right|_{T=T_{C}}$ polarization sensitivity $\frac{d P}{d T}$ at Curie temperature $\mathrm{T}_{\mathrm{c}}$ and

$$
C=\frac{P_{i}+P_{f}}{2}-B T_{C}
$$

Eq. (1) was determined by the physical mechanism that the dipole-ordered state can be enhanced by decreasing temperature. A result of this model, the electrocaloric entropy change caused by variation of the external electric field from $\mathrm{E} 1$ to $\mathrm{E} 2$ is given by

$$
\Delta S^{E}=\left(-A\left(\frac{P_{i}-P_{f}}{2}\right) \sec h^{2}\left(A\left(T_{C}-T\right)\right)+B\right) \Delta E
$$

The result derived from Eq. (4) is the maximum entropy (where $\mathrm{T}=\mathrm{Tc}$ ) that can be expressed as:

$$
\Delta S_{\text {Max }}^{E}=\left(-A\left(\frac{P_{i}-P_{f}}{2}\right)+B\right) \Delta E
$$

Full width half maximum (FWHM) can be deduced by the difference between two extreme values of the independent variables at which the dependent variable is equal to half of its maximum value.

$$
\delta T_{F W H M}=\frac{2}{A} \cosh ^{-1}\left(\sqrt{\frac{2 A\left(P_{i}-P_{f}\right)}{A\left(P_{i}-P_{f}\right)+2 B}}\right)
$$

The above equation gives full width at half maximum entropy variation of a given material.

Electrocaloric cooling power can by obtain by $-\Delta S_{\text {Max }}$ and $\delta \mathrm{T}_{\mathrm{FWHM}}$. The product of these two parameters gives relative cooling power (RCP).
$R C P=-\Delta S_{M}(T, \Delta E) \times \delta T_{F W H M}$

$$
=\left(P_{i}-P_{f}-2 \frac{B}{A}\right) \Delta E \times \cosh ^{-1}\left(\sqrt{\frac{2 A\left(P_{i}-P_{f}\right)}{A\left(P_{i}-P_{f}\right)+2 B}}\right)
$$

Polarization related change of heat capacity is given is given by

$$
\Delta C_{P, E}=T \frac{\delta \Delta S_{E}}{\delta T}
$$

From eq (4) and eq (8)

$$
\Delta C_{P, E}=-2 A^{2}\left(\frac{P_{i}-P_{f}}{2}\right) \sec h^{2}\left(A\left(T_{C}-T\right)\right) \tanh \left(A\left(T_{C}-T\right) \Delta E\right.
$$

A temperature change of a polar system under adiabatic electric field variation from an initial value $\mathrm{E}_{1}$ to final value $\mathrm{E}_{2}$ can be written in the form

$$
\Delta T=-\frac{T}{C_{E \rho}} \int_{E_{1}}^{E_{2}}\left(\frac{\partial P}{\partial T}\right)_{E} d E
$$

From Equations (4) and (10), $\Delta \mathrm{T}$ can be rewritten as

$$
\begin{aligned}
& \Delta T=\frac{A T\left(P_{i}-P_{f}\right)}{2 C_{E \rho}}\left[\operatorname{sech}^{2}\left(A\left(T_{C}-T\right)\right)+B\right] \int_{E_{1}}^{E_{2}} d E \\
& \Delta T=\frac{A T\left(P_{i}-P_{f}\right)}{2 C_{E \rho}}\left[\operatorname{sech}^{2}\left(A\left(T_{C}-T\right)\right)+B\right] \Delta E
\end{aligned}
$$

where is a mass density of NBT-ST and CE a heat capacity per mole at constant electric field.

From these equations $\Delta \mathrm{S}_{\mathrm{Max}}, \delta \mathrm{T}_{\mathrm{FWHM}}, \mathrm{RCP}, \Delta \mathrm{C}_{\mathrm{P}, \mathrm{E}}$ and $\Delta \mathrm{T}$ for NBT-ST easily calculated.
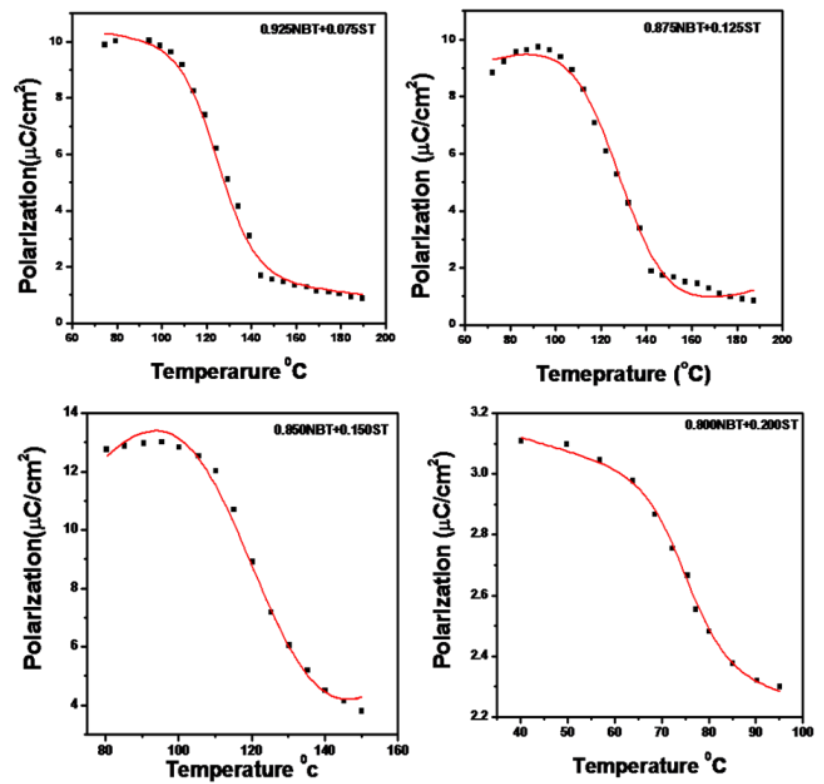

Fig. 3. Temperature dependence of polarization of NBSTA, NBSTB, NBSTC, NBSTD samples. Dots represent the experimental data and line represents the fitted curve.

In Fig. 3 the dots indicate the experimental values obtained for the samples. The solid line in the figures indicates the fitted curve. At the transition temperature, the spontaneous polarization leads to the formation of surface 


\section{Advanced Materials Letters https://aml.iaamonline.org}

charges and stray charges accumulate on the surface of ferroelectric material. When there is a nonhomogeneous distribution of spontaneous polarisation, the surface charge produces an electric field in the opposite direction of the spontaneous polarisation, known as a depolarization field. The experimental and fitted data is good agreement with each. Initial polarization value, final polarization value and transition temperature of the samples are listed in Table 2.

Table 2. Transition temperature and loop area of NBST samples.

\begin{tabular}{ccccccc}
\hline Sample & $\begin{array}{l}\mathbf{T}_{\mathbf{F E - A F E}} \\
\left({ }^{\mathbf{0}} \mathbf{C}\right)\end{array}$ & $\begin{array}{c}\mathbf{T}_{\text {AFE- }} \\
\mathbf{P E}\left({ }^{\mathbf{0}} \mathbf{C}\right)\end{array}$ & $\begin{array}{l}\text { Loop area } \\
\left(\mathbf{k V}-\boldsymbol{\mu C} / \mathbf{c m}^{\mathbf{3}}\right)\end{array}$ & $\begin{array}{l}\mathbf{T}_{\mathbf{i}} \\
\left({ }^{\mathbf{0}} \mathbf{C}\right)\end{array}$ & $\begin{array}{c}\mathbf{T}_{\mathbf{f}} \\
\left({ }^{\circ} \mathbf{C}\right)\end{array}$ & $\begin{array}{c}\mathbf{T}_{\mathbf{C}} \\
\left({ }^{\circ} \mathbf{C}\right)\end{array}$ \\
\hline NBSTA & 195 & 321 & 504 & 74.5 & 189.4 & 133 \\
NBSTB & 198 & 314 & 502 & 82.5 & 187.4 & 127 \\
NBSTC & 200 & 305 & 522 & 80.4 & 150.1 & 120 \\
NBSTD & - & 204 & 167 & 40.1 & 130.3 & 80 \\
\hline
\end{tabular}

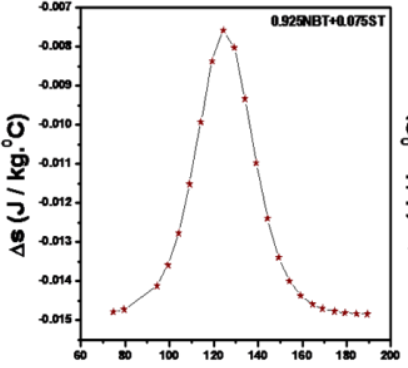

Temperarure ${ }^{\circ} \mathrm{C}$
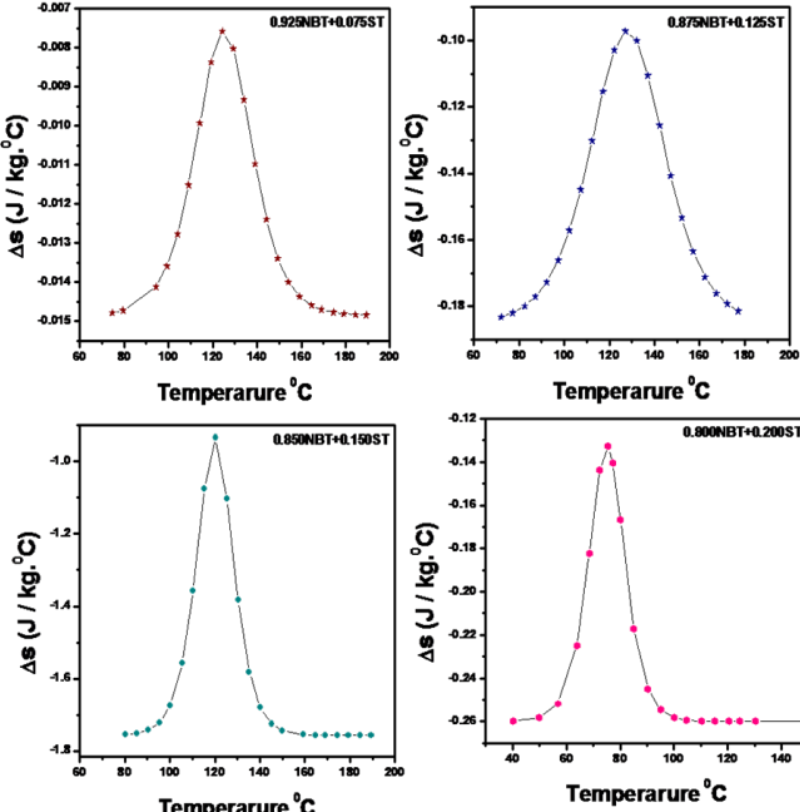

Temperarure ${ }^{\circ} \mathrm{C}$

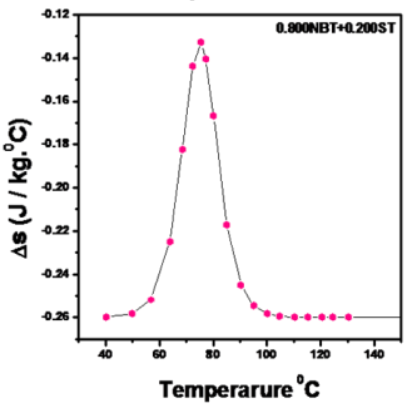

Fig. 4. Variation of entropy with temperature for NBSTA, NBSTB, NBSTC, NBSTD samples.

Fig. 4 indicates the variation of entropy with temperature of NBT-ST sample. Maximum variation is observed near the transition temperature. FE-AFE transition in NBT-ST ceramics has transition near $150^{\circ} \mathrm{C}$ $180^{\circ}[\mathbf{1 4}]$. By increasing the applied electric field, the dipole ferroelectric ordered state can be improved. As a result, when the field is applied, the entropy reduces and the ferroelectric material releases heat, whereas when the field is removed, the entropy rises, and the material absorbs heat. This entropy change within the sample enables for the variations in the temperature and cooling effect within the sample. However, maximum observed in $\Delta S$ vs temperature gives the continuous reorientation of dipoles in the system. If the temperature is increased in the samples, the change of entropy $\Delta \mathrm{S}$ is compensated by an increase of

the lattice vibrations. Maximum entropy values of NBST samples are listed in Table 3. If we observe the table, the transition temperatures of the samples from FE-AFE and AFE-PE are found little higher than what the values obtain in the entropy variations. This may due to the field induced phase transitions in the samples.

Table 3. Calculated values of NBT-ST sample.

\begin{tabular}{cccccccc}
\hline Sample & $\mathbf{A}$ & $\mathbf{B}$ & $\mathbf{C}$ & $\begin{array}{c}\Delta \mathbf{S}_{\mathbf{M a x}}{ }^{*} \mathbf{1 0} \mathbf{2}^{-2} \\
(\mathbf{J} / \mathbf{k g} . \mathbf{C})\end{array}$ & $\begin{array}{c}\Delta \mathbf{T}_{\text {Max }} \\
\left({ }^{\mathbf{0}} \mathbf{C}\right)\end{array}$ & $\begin{array}{c}\boldsymbol{\delta} \mathbf{T}_{\mathbf{F W H M}} \\
\left({ }^{\mathbf{0}} \mathbf{C}\right)\end{array}$ & $\begin{array}{c}\text { RCP } \\
(\mathbf{J} / \mathbf{k g . C})\end{array}$ \\
\hline NBSTA & 0.0592 & -0.0096 & 6.9383 & 0.07 & 0.06 & 30.73 & 0.2326 \\
NBSTB & 0.0454 & -0.0243 & 1.8077 & 0.97 & 0.89 & 42.07 & 4.0591 \\
NBSTC & 0.0920 & -0.0122 & 10.203 & 1.13 & 1.04 & 19.41 & 2.2083 \\
NBSTD & 0.1129 & -0.0044 & 2.996 & 1.06 & 1.01 & 17.28 & 0.1986 \\
\hline
\end{tabular}

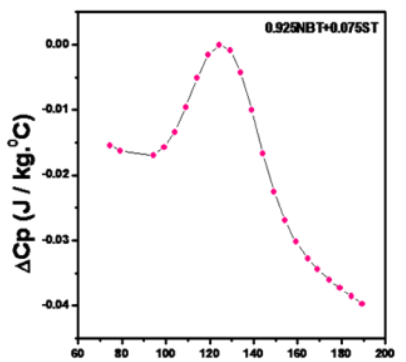

Temperarure ${ }^{\circ} \mathrm{C}$
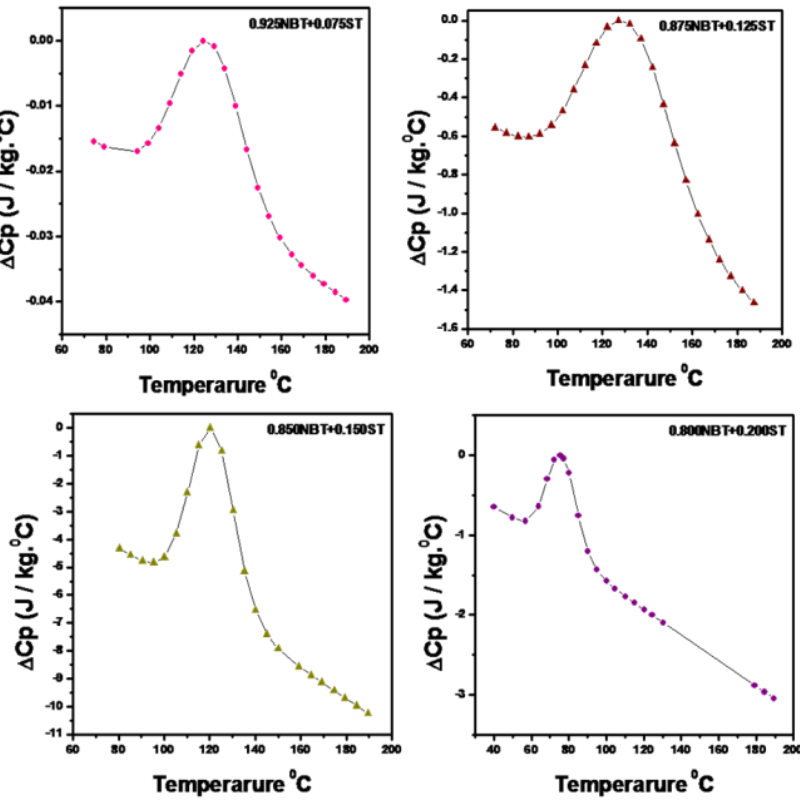

Temperarure ${ }^{\circ} \mathrm{C}$

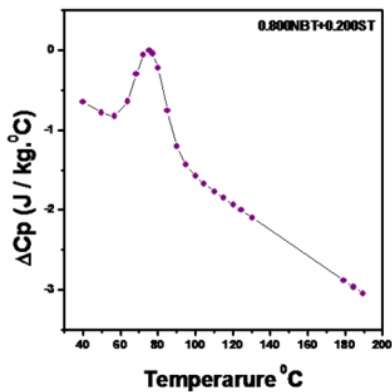

Fig. 5. Change in heat capacity of NBSTA, NBSTB, NBSTC, NBSTD samples.

Fig. 5 shows the variation of heat capacity of the NBSTA-NBSTD samples with temperature. For NBSTANBSTC the variation in heat capacity is observed in the temperature range $110^{\circ} \mathrm{C}-160^{\circ} \mathrm{C}$ but for NBSTD sample it obtained at below $110^{\circ} \mathrm{C}$. These temperatures reflect in the entropy variation curves which indicate FE-AFE transition temperatures of the samples. From Table $\mathbf{3}$ it can be observe that the $\delta \mathrm{T}_{\text {FWHM value of the samples increasing as }}$ ST content in the samples is increasing. But for NBSTD sample show decreased value the reason for this is induced paraelectric behavior of the sample due to high ST paraelectric phase [15]. Relative cooling power of the sample is also showing the same scenario as variations obtained for $\delta \mathrm{T}_{\mathrm{FWHM}}$ values. By comparing all the parameters related to electrocaloric effect NBSTB sample showing better values which can be suitable for cooling applications. 


\section{Conclusions}

NBT-ST samples synthesize by sol gel and solid-state route was taken to observe the polarization and entropy variations. Electrocaloric effect and entropy variation was observed by fitting data which is obtained from P-E loops. Maximum entropy variations observed near FE-AFE transition temperatures of the samples. Relative cooling power observed in the sample was preferably good for refrigeration applications. This reduction in operating temperature can be used in cooling systems. The obtained results show NBST samples are yielding good values which can be suitable for applications in on-chip cooling integrated circuits.

\section{Acknowledgments}

The authors thank UGC, Delhi for providing financial assistance.

\section{Keywords}

NBT, ST, entropy change, electrocaloric effect.

Received:

Revised:

Accepted:

\section{References}

1. Mischenko, A. S.; Zhang, Q.; Whatmore, R. W.; et.al, Appl. Phys. Letter., 2006, 89, 242912.

2. Feng, Zuyong; Shi, Dongqi; Dou, Shixue; Solid State Commun. 2011, 151, 123.

3. Cao, Mao-Sheng; Wang, Xi-Xi; Zhang, Min; Shu, Jin-Cheng; et.al, Adv. Mater. Lett., 2019, 1807398.

4. Wang, Dawei; Sheng Cao, Mao; Zhang, Shujun; Journal of the European Ceramic Society, 2012, 32, 433.

5. Mandipalli, Venkata Ramana; Ramamanohar Reddy, N.; Chin Lin, Wen; et.al, Adv. Mater. Lett., 2015, 6, 27

6. Rajani Malathi, A.; Devi, Ch. Sameera; Kumar, G. S.; Vithal, M.; Prasad, G.; Ionics, 2013, 19, 1751.

7. Bai, Yang; Zheng, Guang-Ping; Shi, San-Qiang; Mater. Res. Bull. 2011, 46, 1866.

8. Hagberg, J.; Dunce, M.; Birks, E.; Antonova, M.; Stenbeg, A.; Ferroelectrics, 2012, 428, 20.

9. Valant, Matjaz; Prog. Mater Sci., 2012, 57, 980.

10. Maqbool, Adnan; Rahman, Jamil ur; Hussain, Ali; et.al, IOP Conf. Series: Materials Science and Engineering 2014, 60, 012047.

11. Rajani Malathi, A.; Kumar, G.S.; Prasad, G.; Phase Transitions $\mathbf{2 0 1 5}, 88,169$.

12. Sheng-Guo, LU; Xin-Gui, Tang; Shang-Hua, Wu; Qi-Ming, Zhang; J. Inorg Mater., 2014, 29, 6.

13. Aly Hamad, Mahmoud; Phase Transitions 2012, 85, 159.

14. Tu, C.S.; Siny, I.G.; Schmidt, V.H.; Phys. Rev. B 1994, 49, 115501.

15. Ang, Chen; Bhalla, A. S.; Guo, Ruyan; Cross, L. E.; Appl. Phys. Letter., 2000, 76, 1929. 